Musculoskeletal Sciences, Oxford, United Kingdom; ${ }^{3}$ Karolinska Institutet and Karolinska University Hospital, Department of Molecular medicine and Surgery, Stockholm, Sweden; ${ }^{4}$ Maastricht University Medical Center+, 4 Department of Clinical Pharmacy and Toxicology, Maastricht, Netherlands

Background: Dupuytren disease (DD) is multifactorial, with several genetic and environmental risk factors contributing to disease susceptibility. High body mass index, however, was suggested to be protective of DD. ${ }^{1}$ The impact of weight loss among obese patients on DD has not been assessed to date.

Objectives: To assess the association between bariatric surgery and DD in a secondary care setting.

Methods: We performed a propensity score (PS)-matched cohort study using data from Swedish nationwide healthcare registries (patient registry [secondary care], causes of death registry, prescribed drug registry). Patients aged 30-79 years who underwent bariatric surgery between 2006 and 2019 were matched to up to 2 obese bariatric surgery-free patients (called unexposed patients) based on their PS. PS-matching was carried out in risk set sampling to reduce selection bias, within 4 sequential cohort entry blocks to account for time trend biases. The outcome DD was defined as a diagnosis of DD in secondary care or partial or total fasciotomy of wrist or hand. After a 1-year run-in period, patients were followed in an "as-treated" approach. We applied Cox proportional hazard regression to calculate hazard ratios (HR) with $95 \%$ confidence intervals (Cls) of incident DD among bariatric surgery patients when compared to obese unexposed patients overall, and in subgroups of age, sex, bariatric surgery type, and by duration of follow-up.

Results: A total of 34959 bariatric surgery patients were PS-matched to 54769 obese unexposed patients. A total of $71.6 \%$ of bariatric surgery patients were women. Bariatric surgery patients had a mean age of 45.5 years and a mean follow-up of 6.9 years. All patient characteristics in obese unexposed patients were highly similar. We observed 126 and 136 severe DD cases among bariatric surgery and obese unexposed patients, respectively. The risk of DD was significantly increased in bariatric surgery patients compared to obese unexposed patients (HR $=1.30,95 \% \mathrm{Cl} 1.02-1.65)$. The risk of $\mathrm{DD}$ was higher in women $(\mathrm{HR}=1.36,95 \% \mathrm{Cl}$ $1.00-1.84)$ than in men ( $\mathrm{HR}=1.05,95 \% \mathrm{Cl} 0.70-1.58)$. Age did not modify the risk of DD among bariatric surgery patients compared to obese unexposed patients. Malabsorptive bariatric surgery yielded an increased risk of DD when compared to obese unexposed patients $(\mathrm{HR}=1.33,95 \% \mathrm{Cl} 1.04-1.71)$, while restrictive bariatric surgery yielded a null result. The risk of DD increased with duration of follow-up (>5 years of follow-up: $\mathrm{HR}=1.63,95 \% \mathrm{Cl} 1.14-2.34$, null result in earlier follow-up). Conclusion: Our results suggest that substantial weight loss is associated with a latent increased risk of severe DD in an obese population. This observation further strengthens current evidence that high body mass index is protective against DD. The latency of risk increase of DD after bariatric surgery may suggest that slowly adapting metabolic changes may be part of the mechanism of DD emergence.

REFERENCES:

[1] Hacquebord JH, Chiu VY, Harness NG. The Risk of Dupuytren Surgery in Obese Individuals. J Hand Surg Am. 2017, 42: 149-55.

Acknowledgements: We thank Prof. Dr. Jesper Lagergren (Karolinksa Institutet, Stockholm, Sweden) for hosting Dr. Theresa Burkard for a research stay at the Upper Gastrointestinal Surgery Group and making the data available for use. Furthermore, we thank Dr. Giola Santoni (Karolinksa Institutet, Stockholm, Sweden) for her technical support.

Disclosure of Interests: None declared

DOI: 10.1136/annrheumdis-2021-eular.4067

\section{POS1384 DIFFERENT CLINICAL CHARACTERISTICS OF IGG4- RRD PATIENTS AND NON-IGG4-RRD PATIENTS: A LARGE CHINESE COHORT STUDY}

Z. Wang ${ }^{1}$, L. Jiaxi ${ }^{1}$, Y. Liu ${ }^{1} .{ }^{1}$ Peking University People's Hospital, Department of Rheumatology and Immunology, Beijing, China

Background: IgG4-related disease (IgG4-RD) is an autoimmune disorder and frequently involve multiple organs. The respiratory tract is one of the most frequently involved sites.

Objectives: This study aimed to compare the demographic and clinical characteristics of IgG4-related respiratory disease (IgG4-RRD) and non-IgG4-RRD patients in a large cohort.

Methods: We carried out a retrospective study of 452 cases of IgG4-RD (104 IgG4-RRD patients and 348 non-IgG4-RRD patients) diagnosed at Peking University People's Hospital between 2003 and 2020.

Results: IgG4-RRD patients had an elder age of disease onset and diagnosis. Multiorgan involvement and hypocomplementemia were more common in IgG4-RRD. Besides, the level of ESR, eosinophilia, IgG and IgG4 were higher in IgG4-RRD patients. In IgG4-RRD group, salivary gland, lacrimal gland, lymph nodes, biliary system and kidney were more commonly involved than those in the non-IgG4-RRD group. Also, more numbers of organ involvement and biliary involvement were independent risk factors for the development of respiratory involvement in IgG4-RD patients.

Conclusion: Our study revealed demographic, clinical, laboratory and imaging features of IgG4-RRD patients and the underlying differences in pathogenesis between the two phenotypes, which have important implications for the diagnosis and treatment of the disease.

\section{REFERENCES:}

[1] Morales AT, Cignarella AG, Jabeen IS, Barkin JS, Mirsaeidi M. An update on IgG4-related lung disease. European journal of internal medicine. 2019;66:18-24.

[2] Stone JH, Zen Y, Deshpande V. IgG4-related disease. The New England journal of medicine. 2012;366(6):539-51.

[3] Vasaitis L. IgG4-related disease: A relatively new concept for clinicians. European journal of internal medicine. 2016;27:1-9.

[4] Matsui S, Yamamoto H, Minamoto S, Waseda Y, Mishima M, Kubo K. Proposed diagnostic criteria for IgG4-related respiratory disease. Respiratory investigation. 2016;54(2):130-2.

[5] Cao L, Chen YB, Zhao DH, Shi WF, Meng S, Xie LX. Pulmonary function tests findings and their diagnostic value in patients with IgG4-related disease. Journal of thoracic disease. 2017;9(3):547-54

[6] Wallace ZS, Perugino C, Matza M, Deshpande V, Sharma A, Stone JH. Immu noglobulin G4-related Disease. Clinics in chest medicine. 2019;40(3):583-97.

[7]Matsui S. IgG4-related respiratory disease. Modern rheumatology.2019;29(2):251-6.

[8] Johansson SG, Hourihane JO, Bousquet J, Bruijnzeel-Koomen C, Dreborg S, Haahtela T, et al. A revised nomenclature for allergy. An EAACl position statement from the EAACI nomenclature task force. Allergy. 2001;56(9):813-24

[9] Fei Y, Shi J, Lin W, Chen Y, Feng R, Wu Q, et al. Intrathoracic Involvements of Immunoglobulin G4-Related Sclerosing Disease. Medicine. 2015;94(50):e2150.

[10] Wallace ZS, Deshpande V, Mattoo H, Mahajan VS, Kulikova M, Pillai S, et al. IgG4-Related Disease: Clinical and Laboratory Features in One Hundred Twenty-Five Patients. Arthritis \& rheumatology (Hoboken, NJ). 2015;67(9):2466-75.

[11] Yamada K, Yamamoto M, Saeki T, Mizushima I, Matsui S, Fujisawa Y, et al New clues to the nature of immunoglobulin G4-related disease: a retrospective Japanese multicenter study of baseline clinical features of 334 cases. Arthritis research \& therapy. 2017;19(1):262.

[12] Borges T, Silva S. IgG4-related disease: How to place it in the spectrum of immune-mediated and rheumatologic disorders? Modern rheumatology. 2020;30(4):609-16.

[13] Liu Y, Xue M, Wang Z, Zeng Q, Ren L, Zhang Y, et al. Salivary gland involvement disparities in clinical characteristics of IgG4-related disease: a retrospective study of 428 patients. Rheumatology (Oxford, England). 2020;59(3):634-40.

[14] Matsui S, Taki H, Shinoda K, Suzuki K, Hayashi R, Tobe K, et al. Respiratory involvement in IgG4-related Mikulicz's disease. Modern rheumatology. 2012;22(1):31-9.

Disclosure of Interests: None declared

DOI: 10.1136/annrheumdis-2021-eular.4277

\section{POS1385 AUTOIMMUNE DISEASES ASSOCIATED WITH CHRONIC LIVER DISEASES}

N. Trad ${ }^{1}$, G. Mohamed ${ }^{2}$, B. Ben Slimen ${ }^{1}$, K. Boughoula ${ }^{2}$, S. Bizid ${ }^{1}$, H. Ben Abdallah' ${ }^{2}$, R. Bouali ${ }^{1}$, M. N. Abdelli ${ }^{2}{ }^{1}$ Military Hospital of Tunis, Gastroenterology, Tunis, Tunisia; ${ }^{2}$ Military Hospital of Tunis, Gastroenterology, Tunis, Tunisia

Background: Chronic liver diseases whatever their etiologies could be associated with immunological disturbances.

Objectives: Our aim was to evaluate the prevalence and the characteristics of autoimmune diseases associated with chronic liver diseases.

Methods: We performed a retrospective analysis of data from consecutive patients followed in our department for chronic liver diseases recruited from January 2010 to December 2019. Demographic, clinical, and paraclinical data were collected.

Results: A total of 224 patients were included. The mean age was $61.02 \pm 13.2$ years and the sex-ratio was 1.6. The main etiology of chronic liver diseases was vira infection C (32.1\%) followed by viral infection B $(22.8 \%)$ and non-alcoholic steatohepatitis (21.4\%). The prevalence of autoimmune chronic liver diseases was $7.58 \%$ : autoimmune hepatitis $(\mathrm{AlH})$ in four cases, primary biliary cholangitis (PBC) in huit cases, primary sclerosing cholangitis (PSC) in three cases and overlap syndrome (AlH-PBC) in two cases. Autoimmune diseases were noted in 31 cases (13.9\%): autoimmune hemolytic anemia in 15 cases, autoimmune thyroiditis in 12 cases, one case of psoriasis, one case of CREST syndrome, one case of Sjögren's syndrome and one case of autoimmune thrombocytopenia. Autoimmune pathologies were more associated with autoimmune chronic liver disease than other causes of chronic liver disease ( $47 \%$ vs $11.1 \%, p<0.001)$. Autoimmune pathologies were not statistically associated with female gender $(p=0.085)$ or young age $(p=0.483)$.

Conclusion: In our study, the prevalence of autoimmune diseases during chronic liver diseases was $13.9 \%$. This prevalence was higher in the case of autoimmune chronic liver diseases (47\%), which would underline the importance of systematic screening for clinical and biological immune manifestations in those patients.

Disclosure of Interests: None declared

DOI: 10.1136/annrheumdis-2021-eular.4288 